\title{
Um Modelo de Maturidade para Planejamento Estratégico de SI/TI
}

\author{
José Gilson de Almeida Teixeira Filho ${ }^{1}$, Hermano Perrelli de Moura ${ }^{2}$ \\ ${ }^{1}$ Universidade de Pernambuco - UPE - Caruaru - PE - Brasil \\ ${ }^{2}$ Universidade Federal de Pernambuco - UFPE - Recife - PE - Brasil \\ jgatf@upe.poli.br, hermano@cin.ufpe.br
}

\begin{abstract}
This paper presents a model to assess the maturity level of the strategic IS/IT planning of Brazilian governmental organizations, named MMPE-SI/TI (Gov). It was designed in accordance with the main models and standards used for national and international definition and assessment of processes and consist of a reference model (RM), an assessment method (AM) and a database of best practices (DBP). The MMPE-SI/TI (Gov) model has 5 maturity levels, 6 capacity levels, 16 processes and 124 best practices for strategic IS/IT planning directed to Brazilian governmental organizations.

Resumo: Esse artigo propõe um modelo para avaliar o nível de maturidade do planejamento estratégico de SI/TI de organizações governamentais brasileiras, denominado MMPE-SI/TI (Gov). Ele foi definido em conformidade com os principais modelos e normas nacionais e internacionais utilizados para definição e avaliação de processos e é formado por um modelo de referência (MR), um método de avaliação (MA) e um banco de melhores práticas (BMP). O modelo MMPE-SI/TI (Gov) possui 5 níveis de maturidade, 6 níveis de capacidade, 16 processos e 124 melhores práticas para planejamento estratégico de SI/TI direcionadas às organizações governamentais brasileiras.
\end{abstract}

\section{INTRODUÇÃO}

Hoje, é fato que o número de organizações, pequenas, médias ou grandes, e ainda públicas ou privadas que utiliza SI/TI (Sistemas de Informação/Tecnologia da Informação) é imenso, mas poucas, principalmente no Brasil, elaboram ou seguem verdadeiramente um planejamento estratégico de SI/TI formal [BRASIL, 2008d]. Essas organizações devem criar, manter e seguir um planejamento estratégico de SI/TI alinhado ao planejamento estratégico institucional, além é claro de formalizar o uso de alguma metodologia para guiar todo o processo [IRANI, 2002; REZENDE, 2007a; 2007b].

As organizações também estão à procura de modelos que permitam avaliar o nível de maturidade dos seus processos. Tendo em vista o dinamismo e a complexidade dos negócios, cada dia mais, as organizações estão buscando utilizar SI/TI mais robustos que facilitem o planejamento, controle e execução dos seus projetos para permitir transformar as estratégias em ações que possam aumentar o sucesso dos 
projetos [GALLIERS e LEIDNER, 2009; KERZNER, 2006; WARD e PEPPARD, 2002].

Em 2008 foram apresentados os resultados de uma pesquisa sobre a situação de governança de TI realizada com 255 órgãos/entidades da Administração Pública Federal (APF) brasileira. Observou-se que entre os pesquisados, 47,0\% não mantinham planejamento estratégico institucional em vigor. Esse fato demonstrou que quase metade das organizações pesquisadas não possuía a cultura de planejar estrategicamente suas ações e apenas reagiam às demandas e às mudanças ocorridas no seu âmbito de atuação. Essa forma de atuação dificulta o planejamento das ações de SI/TI. Foram confrontados esses dados com a informação de que $59 \%$ das organizações pesquisadas não faziam planejamento estratégico de SI/TI. Dos 47\% dos órgãos/entidades que afirmaram não possuir planejamento estratégico institucional, 81\%, isto é, 97 órgãos/entidades não possuíam planejamento estratégico de SI/TI. Por outro lado, o fato de existir planejamento estratégico institucional, por si só, não garantiu que haveria planejamento estratégico de SI/TI. Em 40\% das organizações que dispunham do primeiro, não havia o segundo, ver Gráfico 1 [BRASIL, 2008d].

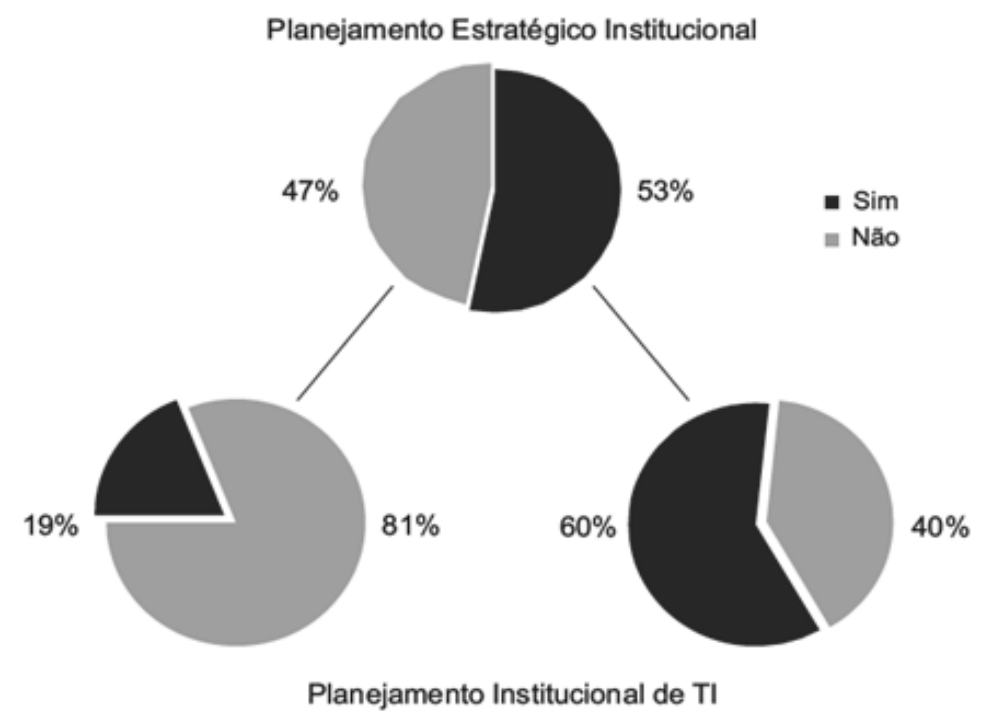

Gráfico 1. Resultado da Pesquisa de 2008 com 255 Órgãos [BRASIL, 2008d].

Em 2010 foram apresentados novos resultados sobre a situação da governança de TI a APF e observou-se que apenas $21 \%$ das instituições não faziam planejamento estratégico institucional, contra os $47 \%$ observados na pesquisa anterior. Isso indicou que houve um crescimento significativo do número de instituições que passaram a fazer o planejamento estratégico institucional. Em relação ao planejamento estratégico de SI/TI, observou-se que $61 \%$ das instituições não faziam planejamento estratégico de SI/TI, contra os 59\% observados na pesquisa anterior, ver Gráfico 2. Isso indicou que houve uma diminuição do número de instituições que faziam planejamento estratégico de SI/TI, o que foi considerado preocupante pelo Tribunal de Contas da União - TCU [BRASIL, 2010]. 


\section{PLANEJAMENTO ESTRATÉGICO}

$\approx 2007 \approx 2010$

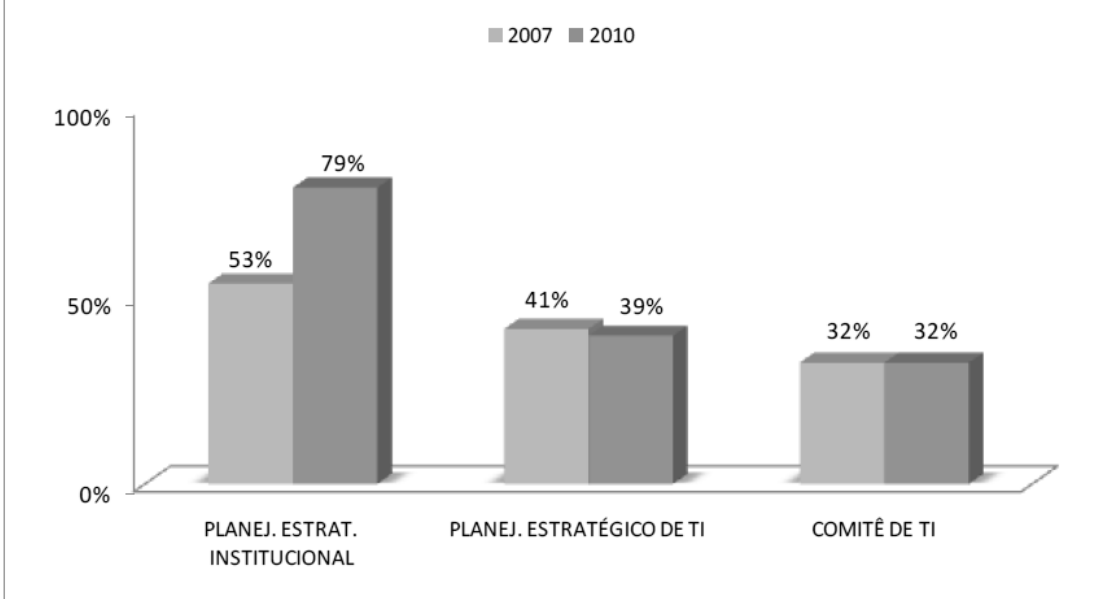

Gráfico 2. Resultado da Pesquisa de 2010 com 265 Órgãos [BRASIL, 2010].

Nesse contexto, observa-se que algumas organizações elaboram seu planejamento estratégico de SI/TI, mas possuem grande dificuldade em avaliar a qualidade e os resultados efetivos ao longo dos anos, e ainda, se o plano foi bem definido e coordenado. As organizações, de maneira geral, desconhecem o quanto estão aderentes às melhores práticas mundiais e dificilmente encontram formas de se comparar (benchmarking) com outras organizações, seja no cenário nacional ou internacional [APPLEBY et al. 2007, CRAWFORD, 2007; OGC, 2008; OGC, 2006; PENNYPACKER, 2005; LI e YE, 1999; PEREZ, 1998; THONG, 1999].

Para auxiliar as organizações a responderem essas questões, foi elaborado o modelo denominado MMPE-SI/TI (Gov) - Modelo de Maturidade para Planejamento Estratégico de SI/TI direcionado às Organizações Governamentais Brasileiras [TEIXEIRA FILHO, 2010].

O MMPE-SI/TI (Gov) foi definido para possibilitar um fácil acesso às melhores práticas mundiais e está em conformidade com os principais modelos e normas nacionais e internacionais utilizados para definição e avaliação de processos, tais como: ISO/IEC 12207, ISO/IEC 15504-1, CMMI, MPS.BR: Guia Geral, COBIT, MMGP, OPM3, PMMM [ISO/IEC 12207, 2008; ISO/IEC 15504-1, 2004, ITGI, 2007; KERZNER, 2005; PMI, 2003; PRADO, 2008; SEI, 2006; SOFTEX, 2009].

Vale destacar que melhores práticas (MP) são visões de organizações e profissionais globais que através da vivência no mercado conseguem perceber práticas, que se utilizadas em outras organizações podem melhorar seu desempenho da mesma forma [CBP, 2006, KERZNER, 2005; PMI, 2009; PMI, 2003]. Já modelo de maturidade é uma estrutura conceitual, composta por processos bem estabelecidos, através do qual uma organização desenvolve-se de modo sistêmico a fim de atingir um estado futuro desejado. A cada degrau alcançado nessa jornada, um modelo de maturidade reconhece e sinaliza o amadurecimento progressivo da organização [CRAWFORD, 2007; KERZNER, 2005; PMI, 2003; PRADO, 2008; SEI, 2006].

Este modelo recebe como entrada as melhores características dos principais modelos/metodologias/autores pesquisados por Teixeira Filho, além de um conjunto de 
melhores práticas de planejamento estratégico de SI/TI (MP.SI/TI), maturidade em gerenciamento de projetos (MP.MGP) e especialmente, governo brasileiro (MP.GOV) [TEIXEIRA FILHO, 2010].

Ele está estruturado em três componentes: Modelo de Referência (MR), Banco de Melhores Práticas (BMP) e Método de Avaliação (MA) e apresenta quatro áreas que auxiliam a organização a direcionar seus esforços para desenvolver o seu planejamento estratégico de SI/TI, são elas: Gestão, Organização, Pessoas e Tecnologia. A Figura 1 apresenta a visão geral do MMPE-SI/TI (Gov) e de todas as suas partes.

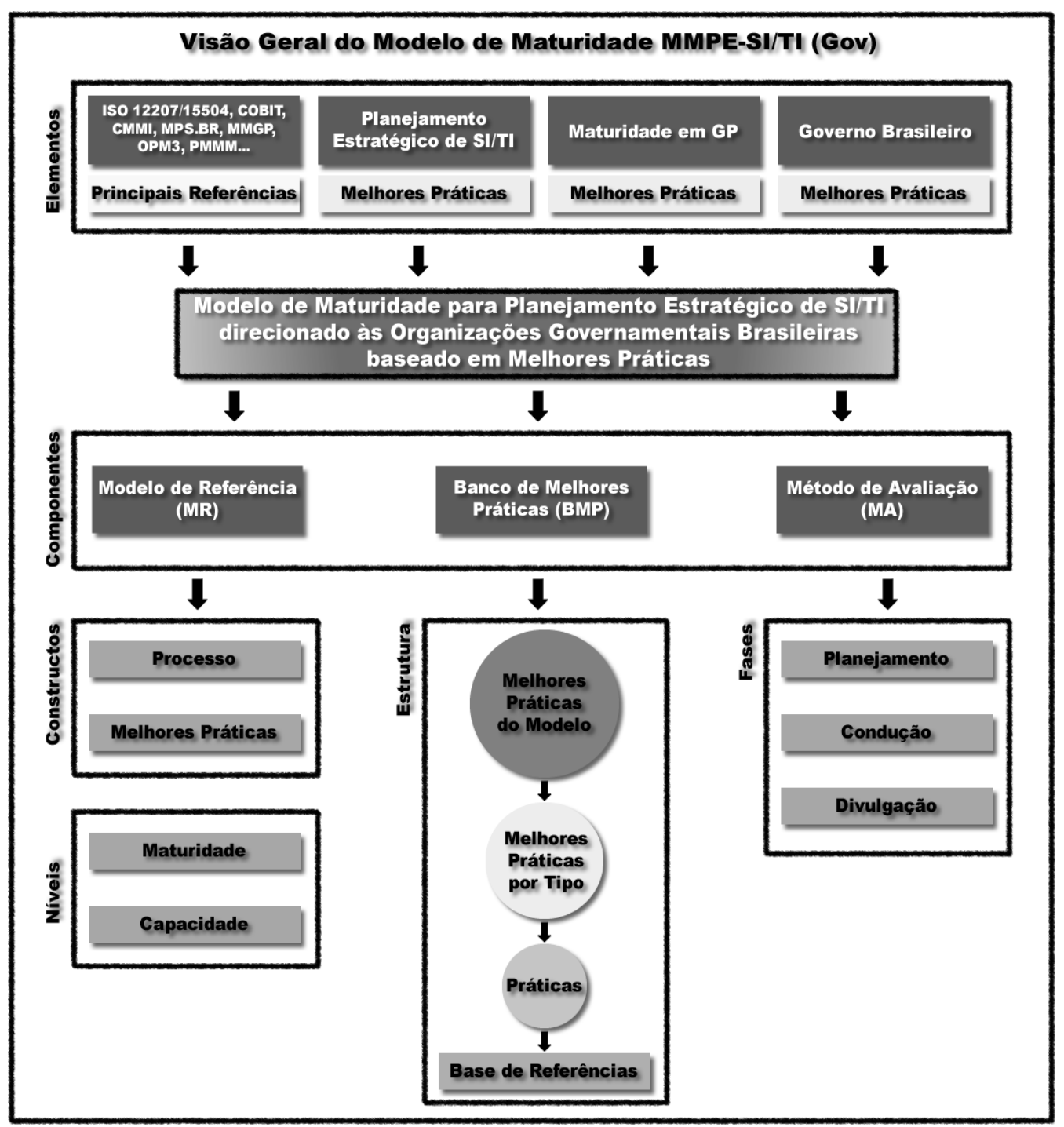

Figura 1. Visão Geral do MMPE-SI/TI (Gov) [TEIXEIRA FILHO, 2010].

O MMPE-SI/TI (Gov) possui 5 níveis de maturidade, 6 níveis de capacidade, 16 processos e 124 melhores práticas para planejamento estratégico de SI/TI direcionadas às organizações governamentais brasileiras (ver Figura 2) [TEIXEIRA FILHO, 2010]. 


\begin{tabular}{|c|c|c|c|}
\hline Nível & Processos & Áreas & Atributos do Processo \\
\hline 1 & $\begin{array}{l}\text { Promover Consciência Estratégica (PCE) } \\
\text { Assegurar Conformidade Governamental (ACG) }\end{array}$ & $\begin{array}{l}\text { Gestão } \\
\text { Organização }\end{array}$ & AP 1.1 \\
\hline 2 & $\begin{array}{l}\text { Gerenciar Recursos Humanos (GRH) } \\
\text { Educar e Treinar Pessoas (ETP) } \\
\text { Gerenciar Projetos (GEP) } \\
\text { Gerenciar Medição e Análise (GMA) }\end{array}$ & $\begin{array}{l}\text { Pessoas } \\
\text { Pessoas } \\
\text { Gestão } \\
\text { Gestão }\end{array}$ & AP $1.1 ;$ AP $2.1 ;$ AP $2.2 ;$ \\
\hline 3 & $\begin{array}{l}\text { Definir o Processo Organizacional (DPO) } \\
\text { Gerenciar Aquisições e Terceirizações (GAT) } \\
\text { Gerenciar Infraestrutura de SI/TI (GIN) } \\
\text { Gerenciar Qualidade (GQA) } \\
\text { Fomentar Gestão do Conhecimento (FGC) }\end{array}$ & $\begin{array}{l}\text { Organização } \\
\text { Organização } \\
\text { Tecnologia } \\
\text { Gestão } \\
\text { Organização }\end{array}$ & $\begin{array}{l}\text { AP } 1.1 ; \text { AP } 2.1 ; \text { AP } 2.2 ; \\
\text { AP } 3.1 ; \text { AP } 3.2 ;\end{array}$ \\
\hline 4 & $\begin{array}{l}\text { Avaliar o Processo Organizacional (APO) } \\
\text { Gerenciar Riscos (GRI) } \\
\text { Gerenciar Integração com o Cidadão (GIC) }\end{array}$ & $\begin{array}{l}\text { Organização } \\
\text { Gestão } \\
\text { Pessoas }\end{array}$ & $\begin{array}{l}\text { AP } 1.1 ; \text { AP } 2.1 ; \text { AP } 2.2 ; \\
\text { AP } 3.1 ; \text { AP } 3.2 ; \text { AP } 4.1 ; \\
\text { AP } 4.2 ;\end{array}$ \\
\hline 5 & $\begin{array}{l}\text { Melhorar o Processo Organizacional (MPO) } \\
\text { Otimizar a Gestão Organizacional (OGO) }\end{array}$ & $\begin{array}{l}\text { Organização } \\
\text { Gestão }\end{array}$ & $\begin{array}{l}\text { AP } 1.1 ; \text { AP } 2.1 ; \text { AP } 2.2 \text {; } \\
\text { AP } 3.1 ; \text { AP } 3.2 \text { AP } 4.1 ; \\
\text { AP } 4.2 ; \text { AP } 5.1 \text { AP } 5.2 ;\end{array}$ \\
\hline
\end{tabular}

Figura 2. Níveis de Maturidade do Modelo e seus Processos [TEIXEIRA FILHO, 2010].

Este artigo tem como objetivo principal apresentar, em detalhes, apenas um dos processos definidos pelo MMPE-SI/TI (Gov). O processo selecionado foi "Fomentar Gestão do Conhecimento (FGC)" que contém 1 propósito, 3 resultados esperados, 6 melhores práticas e 6 produtos de trabalho.

\section{REVISÃO SISTEMÁTICA DA LITERATURA}

Este artigo adotou revisão sistemática e na etapa I foram considerados os critérios de qualidade e as strings de busca [BIOLCHINI et al. 2005; DYBA et al. 2005; KITCHENHAM et al. 2007; LITTELL et al. 2008; MAFRA e TRAVASSOS, 2006; PENNYPACKER, 2005]. Foram obtidos no total 827 estudos (ver Figura 3).

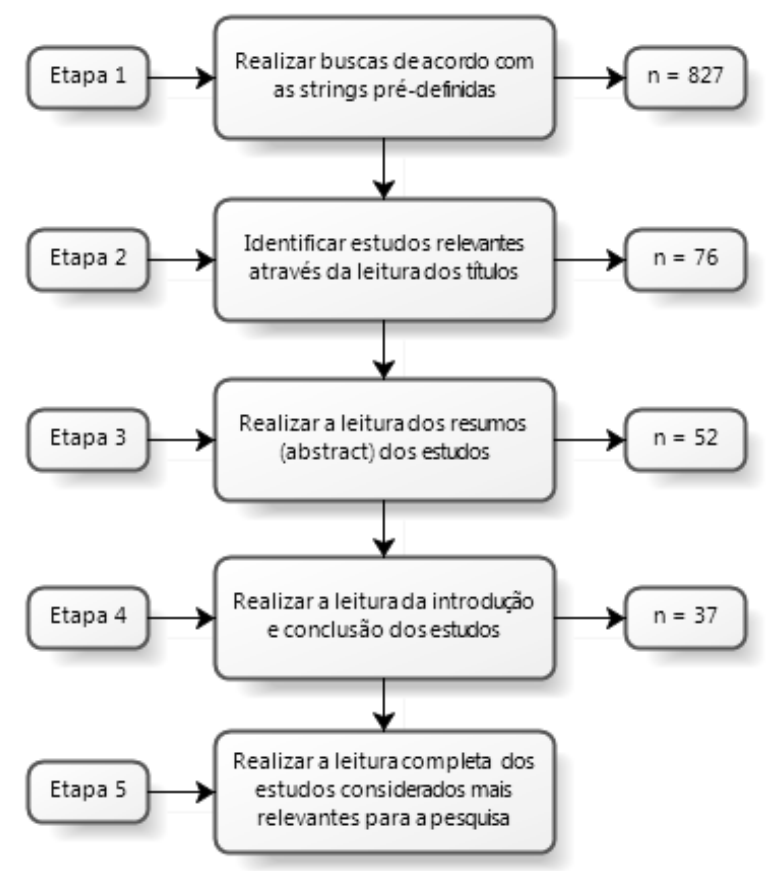

Figura 3. Etapas do Processo de Seleção dos Estudos [TEIXEIRA FILHO, 2010]. 
Na etapa II foram lidos os títulos dos estudos e analisados quais estavam disponíveis por completo. Sendo assim, foram selecionados 76 dos 827 estudos resultantes da busca inicial. Na etapa III, a seleção foi aprimorada com base nos critérios de inclusão e exclusão e a partir da leitura do resumo (abstract) foram selecionados 52 dos 76 estudos relatados até então. Na etapa IV, os estudos passaram por uma leitura da introdução e conclusão e foram selecionados 37 dos 52 estudos restantes. A etapa V estabelecia a leitura completa dos estudos selecionados. Essa leitura final foi realizada de acordo com os propósitos estabelecidos pela pesquisa, ou seja, os estudos foram lidos com o intuito de identificar as práticas ideais para planejamento estratégico de SI/TI, maturidade em gerenciamento de projetos e governo brasileiro.

Os estudos considerados mais relevantes para este trabalho foram publicados entre os anos de 1979 e 2009. Os anos de 2005, 2007 e 2008 apresentaram a maior quantidade de estudos relevantes (5 estudos por ano), ver Gráfico 3.

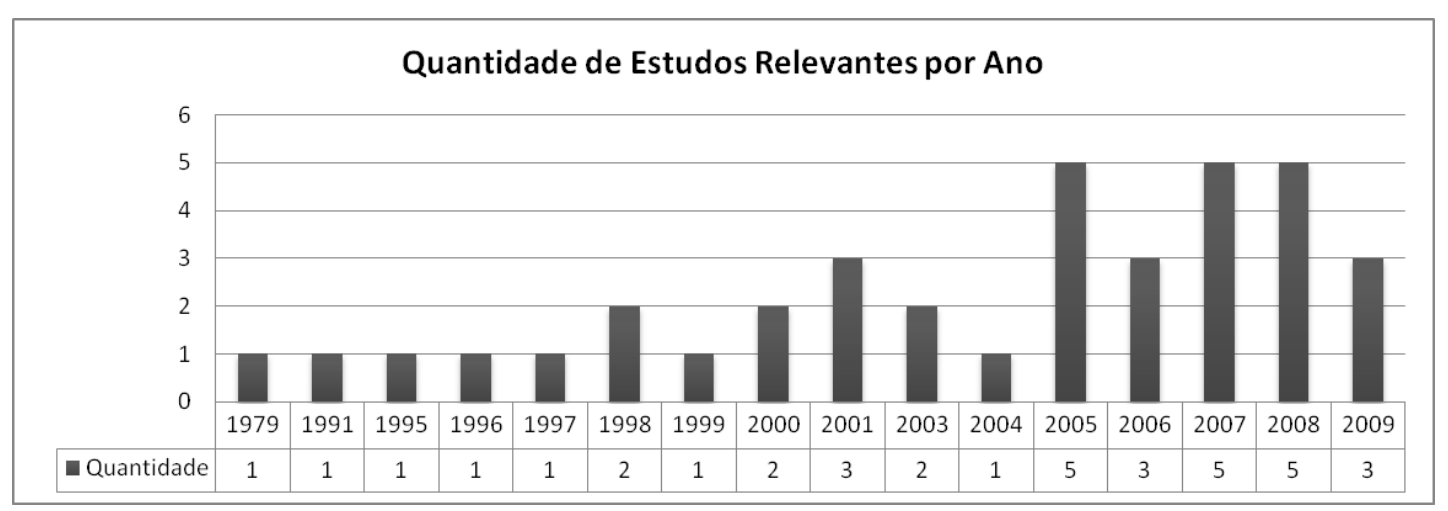

Gráfico 3. Quantidade de Estudos Relevantes por Ano [TEIXEIRA FILHO, 2010].

\section{METODOLOGIA}

Para atender o objetivo central desta pesquisa, foram utilizados diversos procedimentos e técnicas apresentados a seguir:

Quanto ao Método: As investigações, geralmente, utilizam uma combinação de dois ou mais métodos. Neste trabalho adotou-se o método indutivo, estruturalista e comparativo [GIL, 2009; LAKATOS e MARCONI, 2009].

Quanto à Natureza: As pesquisas científicas podem ser classificadas em básica ou aplicada. Neste trabalho foi utilizada a pesquisa aplicada que objetiva gerar conhecimentos para aplicação prática e dirigida à solução de problemas específicos [CERVO e BERVIAN, 2007; SANTOS, 2002].

Quanto aos Procedimentos: Foram realizados os seguintes procedimentos: análise documental, análise bibliográfica baseada em revisão sistemática da literatura, estudos de caso exploratórios, levantamento de campo (survey), estudo de campo baseado em GQM (Goal Question Metric) [BIOLCHINI et al. 2005; DYBA et al. 2005; GIL, 2009; KITCHENHAM et al. 2004; LITTELL et al. 2008; MAFRA e TRAVASSOS, 2006]. 
Quanto às Técnicas de Coleta de Dados: Foram realizadas três entrevistas e aplicados questionários para auxiliar o processo de coleta de dados durante a realização dos estudos de caso exploratórios e durante a realização da validação do modelo junto aos especialistas da área de SI/TI [GIL, 2009; LAKATOS e MARCONI, 2009].

Quanto à Abordagem: Adotou-se duas abordagens: quantitativa e qualitativa. Ambas as abordagens serviram para analisar e discutir os resultados dos estudos de caso exploratórios e do levantamento realizado junto aos especialistas da área de SI/TI [GIL, 2009; SANTOS, 2002].

Quanto à Realização: Utilizou-se o trabalho de campo como estratégia para atingir os objetivos pretendidos, neste caso a pesquisa é realizada em um ambiente onde as variáveis não são controladas, o pesquisador se envolve no ambiente, observa e analisa as variáveis e características da realidade existente [GIL, 2009; SANTOS, 2002].

Quanto à Perspectiva: Para Santos a perspectiva da pesquisa representa qual o campo de interesse que foi focado na pesquisa. Neste caso foram considerados dois campos de pesquisa: Ciências da Computação e Ciências da Administração [SANTOS, 2002].

\section{RESULTADOS}

Um conjunto de melhores práticas e o modelo de referência (MR) foram estabelecidos e validados junto aos especialistas da área de SI/TI com base no GQM. As opiniões foram levantadas por meio de survey utilizando questionários. Essas respostas foram analisadas e interpretadas a fim de demonstrar a importância, capacidade, confiabilidade e coerência, tanto das melhores práticas quanto do modelo de maturidade para planejamento estratégico de SI/TI, que também incluiu a validação dos propósitos e resultados esperados para cada um dos processos existentes no modelo, além de proporcionar as devidas correções e/ou melhorias propostas pelos especialistas. Um banco de melhores práticas (BMP) e um método de avaliação (MA) também foram estabelecidos para o modelo, este último não foi detalhado neste trabalho [TEIXEIRA FILHO, 2010].

\section{1. BANCO DE MELHORES PRÁTICAS (BMP)}

O BMP definido para o MMPE-SI/TI (Gov), busca auxiliar as organizações governamentais brasileiras a atingir um alto grau de maturidade em relação ao planejamento estratégico de SI/TI, a partir da utilização de um conjunto de melhores práticas. Todas elas seguem uma padronização única, que é composta pelas seguintes partes: MP_ID, MP_NOME, MP_DESCRIÇÃO, MP_ID_REF e MP_ÁREA. A base para concepção do BMP envolveu um processo estruturado em cinco etapas, ver Figura 4. 


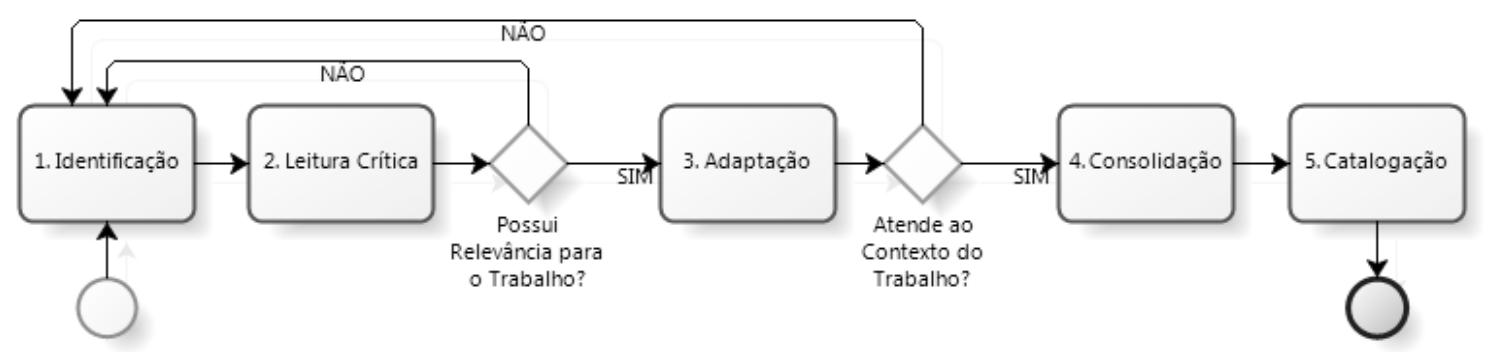

Figura 4. Etapas para Estruturação das Melhores Práticas [TEIXEIRA FILHO, 2010].

Foram identificadas inicialmente, um conjunto de 505 práticas envolvendo as três áreas: planejamento estratégico de SI/TI, maturidade em gerenciamento de projetos e governo brasileiro. A partir deste conjunto inicial de práticas, foi estabelecido um conjunto de 80 melhores práticas que representam as visões unificadas de diversas organizações e profissionais espalhados ao redor do mundo. Deste conjunto de 80 melhores práticas, 32 (40\%) são de planejamento estratégico de SI/TI, 30 (37\%) são de maturidade em gerenciamento de projetos e 18 (23\%) são específicas do governo brasileiro, ver Gráfico 4. Algumas dessas melhores práticas foram divididas/desmembradas para dar mais coerência e lógica contextual ao modelo.

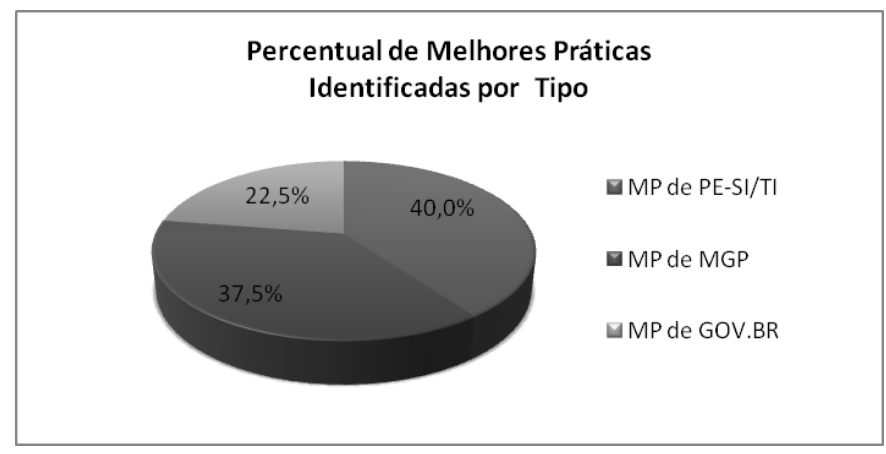

\section{Gráfico 4. Percentual de Melhores Práticas Identificadas por Tipo [TEIXEIRA FILHO, 2010].}

Após esses ajustes, foi estabelecido o banco de melhores práticas (BMP) para o modelo que contém um total de 124 melhores práticas para planejamento estratégico de SI/TI direcionado às organizações governamentais brasileiras. Deste total, 6 melhores práticas estão associadas diretamente à área de gestão do conhecimento, representada neste modelo, pelo processo Fomentar Gestão do Conhecimento (FGC). De acordo com o objetivo proposto por este artigo, apenas o processo FGC será descrito em detalhes.

\section{2. FOMENTAR GESTÃO DO CONHECIMENTO (FGC)}

Este processo faz parte do Modelo de Referência (MR) do MMPE-SI/TI (Gov) e foi definido a partir de um extenso estudo baseado em revisão sistemática da literatura e encontra-se em conformidade com os principais modelos e normas nacionais e internacionais como: ISO/IEC 12207, MPS.BR: Guia Geral, COBIT, Art $4^{\circ}$ do Decreto $n^{0}$ 4.553, de 27 de dezembro de 2002 e Acórdão 1603/2008 TCU Plenário [BRASIL, 2008c; BRASIL, 2002; ISO/IEC 12207, 2008; ITGI, 2007; SOFTEX, 2009]. 
Propósito: Assegurar que o conhecimento individual, as informações e as habilidades sejam coletadas, compartilhadas, reutilizadas e melhoradas por toda a organização.

\section{Resultados Esperados (RE):}

- FGC-RE-01: Uma estratégia adequada de gestão do conhecimento é selecionada;

- FGC-RE-02: A infraestrutura para o compartilhamento de informação comum e específica de toda a organização é estabelecida e mantida;

- FGC-RE-03: O conhecimento é prontamente armazenado e compartilhado por toda a organização.

\section{Melhores Práticas (MP):}

- FGC-MP-01: Desenvolver uma Estratégia de Gestão do Conhecimento [RE: 1];

- FGC-MP-02: Estabelecer um Sistema de Gestão do Conhecimento [RE: 2, 3];

- FGC-MP-03: Criar uma Rede de Colaboradores do Conhecimento [RE: 2, 3];

- FGC-MP-04: Capturar o Conhecimento [RE: 3];

- FGC-MP-05: Disseminar o Conhecimento [RE: 3];

- FGC-MP-06: Melhorar o Conhecimento [RE: 1, 2, 3].

\section{Produtos de Trabalho (PT):}

- FGC-PT-01: Necessidades do negócio [RE: 1];

- FGC-PT-02: Estratégia de gestão do conhecimento [RE: 1].

- FGC-PT-03: Repositório de conhecimento [RE: 2];

- FGC-PT-04: Item de conhecimento [RE: 3];

- FGC-PT-05: Dados sobre o uso dos ativos [RE: 3];

- FGC-PT-06: Registro de comunicação [RE: 3].

Abaixo observa-se uma lista completa das melhores práticas para o processo "Fomentar Gestão do Conhecimento (FGC)". Cada melhor prática apresenta as seguintes informações: MP_ID, MP_NOME, MP_DESCRIÇÃO, MP_ID_REF e MP_ÁREA, ver Figura 5.

\begin{tabular}{|c|c|c|c|c|}
\hline MP_ID & MP_NOME & MP DESCRIÇÃO & MP_ID_REF & MP_ÁREA \\
\hline FGC-MP-01 & $\begin{array}{l}\text { Desenvolver uma } \\
\text { Estratégia de Gestão do } \\
\text { Conhecimento. }\end{array}$ & $\begin{array}{l}\text { A organização deve definir uma estratégia de gestão do conhecimento adequada } \\
\text { que esteja baseada no contexto organizacional, individual, de domínio e de } \\
\text { projeto. [RE: 1]; }\end{array}$ & (MP.SI/TI.N3.04), (MP.MGP.N3.03) & GES \\
\hline FGC-MP-02 & 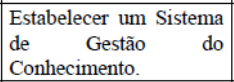 & $\begin{array}{l}\text { A organização deve estabelecer e manter uma infraestrutura de gestão do } \\
\text { conhecimento e um mecanismo para apoiar as atividades de forma a identificar, } \\
\text { classificar, trocar e utilizar os ativos de conhecimento. [RE: } 2,3] \text {; }\end{array}$ & $\begin{array}{l}\text { (MP.SI/TI.N3.02), (MP.MGP.N3.03), } \\
\text { (MP.GOV.N2.01), (MP.GOV.N2.03) }\end{array}$ & ORG \\
\hline FGC-MP-03 & $\begin{array}{|ll|}\text { Criar uma Rede } & \text { de } \\
\text { Colaboradores } & \text { do } \\
\text { Conhecimento. } & \\
\end{array}$ & $\begin{array}{l}\text { A organização deve estabelecer uma rede de especialistas que interaja entre si. } \\
\text { [RE: } 2,3] \text {; }\end{array}$ & $\begin{array}{l}\text { (MP.SI/TI.N1.05), (MP.MGP.N3.03), } \\
\text { (MP.GOV.N3.02) }\end{array}$ & PES \\
\hline FGC-MP-04 & $\begin{array}{l}\text { Capturar } \\
\text { Conhecimento. }\end{array}$ & $\begin{array}{l}\text { A organização deve identificar e registrar cada item de conhecimento de acordo } \\
\text { com o esquema de classificação e os critérios de ativos definidos. [RE: } 3 \text { ]; }\end{array}$ & $\begin{array}{l}\text { (MP.SI/TI.N1.05), (MP.SI/TI.N3.04), } \\
\text { (MP.GOV.N3.02) }\end{array}$ & TEC \\
\hline FGC-MP-05 & \begin{tabular}{|l|} 
Disseminar \\
Conhecimento.
\end{tabular} & $\begin{array}{l}\text { A organização deve compartilhar os ativos de conhecimentos com especialistas, } \\
\text { usuários e projetos. [RE: } 3 \text {; }\end{array}$ & $\begin{array}{l}\text { (MP.SI/TI.N3.04), (MP.MGP.N3.03), } \\
\text { (MP.GOV.N2.03), (MP.GOV.N3.02) } \\
\end{array}$ & TEC \\
\hline FGC-MP-06 & $\begin{array}{l}\text { Melhorar } \\
\text { Conhecimento. }\end{array}$ & $\begin{array}{l}\text { A organização deve validar e enriquecer os ativos de conhecimento para garantir } \\
\text { a sua pertinência e valor para a organização com o passar do tempo. [RE: } 1,2 \text {, } \\
\text { 3]. }\end{array}$ & $\begin{array}{l}\text { (MP.SI/TI.N5.02), (MP.MGP.N3.03), } \\
\text { (MP.MGP.N5.02), (MP.GOV.N5.02) }\end{array}$ & ORG \\
\hline
\end{tabular}


Figura 5. Melhores Práticas FGC [TEIXEIRA FILHO, 2010].

\section{3. VALIDAÇÃO DO PROCESSO "FGC"}

Esta validação baseou-se no GQM e para tanto foram definidas 4 questões e 5 métricas. Essas métricas estabeleceram a forma com que seriam analisadas as respostas. Neste caso uma das métricas baseou-se na escala de likert, enquanto as demais basearam-se em quatro critérios: importância, capacidade, confiabilidade e coerência. Este estudo foi realizado no período compreendido entre 28/07/2010 e 23/08/2010 e obteve a participação de 106 respondentes válidos, o que representa uma amostra significativa da população brasileira, uma vez que este estudo utilizou a amostragem probabilística do tipo estratificada proporcional que esperava obter no mínimo 104,76 respondentes válidos [CERVO e BERVIAN, 2007; GIL, 2009; HONG, 2009; LAKATOS e MARCONI, 2009].

Para os 106 especialistas de SI/TI, este processo apresentou como maiores concentrações percentuais em cada critério, os seguintes valores: totalmente importante (46,9\%), totalmente capaz (38,2\%), totalmente confiável $(38,2 \%)$ e totalmente coerente (38\%). Diante dos resultados apresentados, pode-se constatar que, na média, 73,1\% dos respondentes consideraram este processo como sendo "muito" e "totalmente" importante, capaz, confiável e coerente para todos os critérios, ver Gráfico 5.

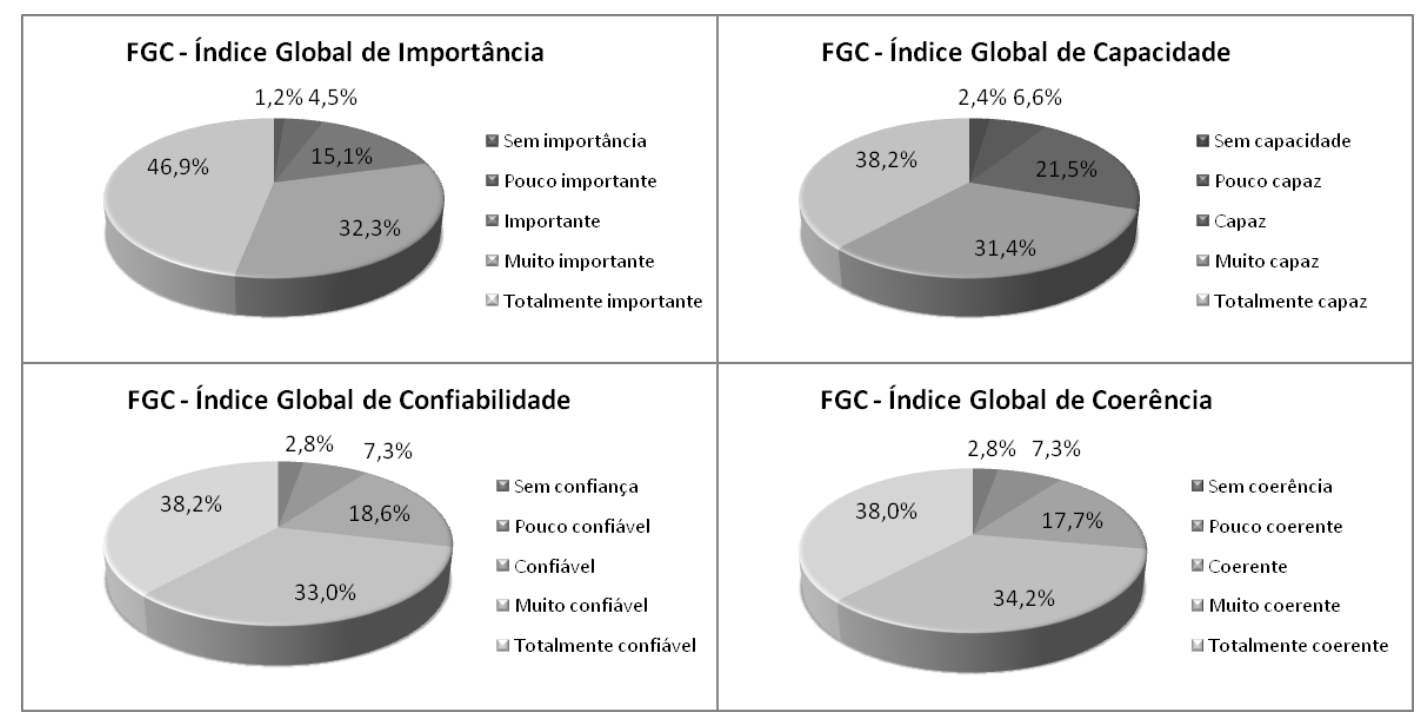

Gráfico 5. FGC - Índices Globais de Desempenho [TEIXEIRA FILHO, 2010].

As variáveis consideradas pelos 106 especialistas como melhores avaliadas na categoria “totalmente” para os quatro critérios foram (ver Gráfico 6):

- FGC-RE-03 para o critério de "importância” com 50,9\% das opiniões, que trata do armazenamento e compartilhamento do conhecimento por toda a organização;

- FGC-RE-02 para os critérios de “capacidade” e “coerência” com respectivamente $41,5 \%$ e $42,5 \%$ das opiniões, que trata de estabelecer e manter a infraestrutura para o compartilhamento de informação comum e específica de toda a organização; e 
- FGC-Propósito para o critério de "confiabilidade" com 39,6\% das opiniões, que trata de assegurar que o conhecimento individual, as informações e as habilidades sejam coletadas, compartilhadas, reutilizadas e melhoradas por toda a organização.

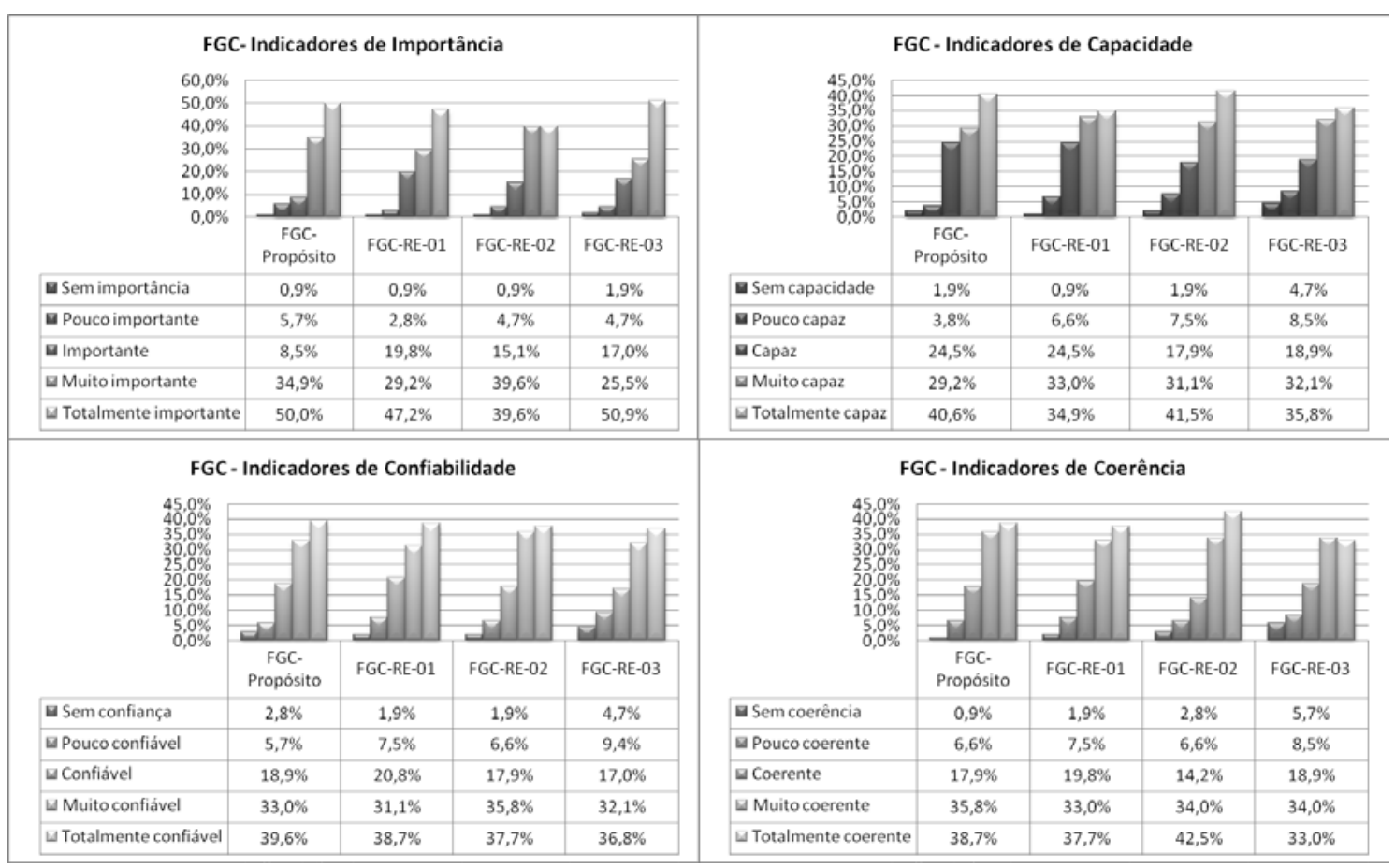

Gráfico 6. FGC - Indicadores de Desempenho [TEIXEIRA FILHO, 2010].

Um dos comentários realizados pelos especialistas de SI/TI sobre este processo é apresentado a seguir:

"RE3: Não armazenado, mas traduzido em atualização de ativos de processos. Faltou: A capacitação imediata da organização frente a mudanças do ativo de processos é assegurado [...] “ (E13).

Com relação ao comentário do especialista (E13), o modelo estabelece duas melhores práticas que respondem bem esse comentário: "FGC-MP-05: Disseminar o Conhecimento", onde a organização deve compartilhar os ativos de conhecimentos com especialistas, usuários e projetos, isto já inclui os ativos de processos e o "FGC-MP-06: Melhorar o Conhecimento”, onde a organização deve validar e enriquecer os ativos de conhecimento para garantir a sua pertinência e valor para a organização com o passar do tempo [BRASIL, 2009d; CASSIDY, 2005; CURRY e FERGUSON, 2000; GOYAL, 2007; GROVER e SEGARS, 2005; HONG, 2009; KUNNATHUR e SHI, 2001; MENTZAS, 1997; NEWKIRK e LEDERER, 2006; PHILIP, 2007; PRADO, 2008; TEUBNER, 2007].

\section{CONCLUSÕES}

Ao longo do trabalho, foi possível verificar a importância do tema "planejamento estratégico de SI/TI" para as organizações governamentais brasileiras, ao mesmo tempo que foi possível observar as suas principais deficiências. Percebeu-se que ao investir na busca pela excelência na maturidade, as organizações devem utilizar as melhores 
práticas mundiais para proporcionar um melhor gerenciamento e conseqüentemente uma evolução mais rápida, gradual e sustentável do nível de maturidade/capacidade. O planejamento estratégico de SI/TI é uma importante função gerencial, que pode ajudar uma organização a usar SI/TI mais competitivamente, identificar novas aplicações de SI/TI e prever melhor as necessidades de recursos [BASU, 2002; LUFTMAN et al. 2005; SALMELA et al. 2000; TEUBNER, 2007].

Esse tema é por natureza bastante complexo e subjetivo. Isso pode ser facilitado através de avaliações periódicas que capturem a visão da indústria e busquem novas motivações para melhoria contínua dos processos. Essas análises podem ser realizadas tanto com relação às descrições do nível de maturidade como um todo, ou ainda com maior rigor, tratando cada um dos processos individualmente. De qualquer forma, é inevitável a adoção de um processo de avaliação e análise da maturidade, principalmente, aquelas organizações que pretendem se destacar no seu âmbito de atuação [CASSIDY, 2005; KERZNER, 2005].

Nesse contexto aplica-se o MMPE-SI/TI (Gov) que é um modelo de maturidade para planejamento estratégico de SI/TI direcionado às organizações governamentais brasileiras baseado em melhores práticas, criado como resultado de uma tese de doutorado [TEIXEIRA FILHO, 2010].

Este artigo apresentou, em detalhes, apenas o processo "Fomentar Gestão do Conhecimento (FGC)", para servir de exemplo e facilitar o entendimento dos leitores. O modelo MMPE-SI/TI (Gov) ${ }^{1}$ possui 16 processos no total, não sendo possível apresentá-los completamente nesse trabalho, por questões de espaço. Este processo tem o objetivo de assegurar que o conhecimento individual, as informações e as habilidades sejam coletadas, compartilhadas, reutilizadas e melhoradas por toda a organização.

Na média, 73,1\% dos respondentes afirmaram que o processo "Fomentar Gestão do Conhecimento (FGC)" era "muito" e "totalmente" importante, capaz, confiável e coerente em todos os critérios. Essa informação demonstra o quanto este processo é importante para melhoria da gestão do conhecimento de uma organização governamental brasileira, como também foi considerado amplamente capaz de ser implementado, indicou ser bastante confiável e coerente com o contexto e a realidade deste tipo de organização [TEIXEIRA FILHO, 2010].

Percebe-se, portanto que a gestão do conhecimento é bastante multidisciplinar e pode ser utilizada em praticamente qualquer ambiente ou contexto. Neste caso, utilizouse como parte integrante de um modelo que visa à melhoria da maturidade do planejamento estratégico de SI/TI de organizações governamentais brasileiras.

\section{REFERÊNCIAS}

APPLEBY, T.; CABANIS-BREWIN, J.; CRAWFORD, J. K.; CRUZ, F.; PENNYPACKER, J. S.; WEST, J. L.; WHITE, K. R. J. Advancing Organizational Project Management Maturity. USA: CBP - Center for Business Practices, 2007.

\footnotetext{
${ }^{1}$ http://www.gilsonteixeira.com/mmpe/
} 
BASU, V. The Impact of Organizational Commitment, Senior Management Involvement, and Team Involvement on Strategic Information Systems Planning. Information \& Management, v. 39, p. 513-524, 2002.

BIOLCHINI, J.; MIAN, P. G.; NATALI, A. C.; TRAVASSOS, G. H. Systematic Review in Software Engineering: relevance and utility. Relatório Técnico RT-ES679/05, Programa de Engenharia de Sistemas e Computação (PESC), COPPE/UFRJ, 2005.

BRASIL. ACÓRDÃO 1603/2008 TCU PLENÁRIO. 2008c. Disponível em: < www.mp.go.gov.br/portalweb/hp/12/docs/acordao_tcu_-_13-08-2008.pdf >. Acesso em: 20 de Janeiro de 2009.

BRASIL. DECRETO No 4.553, DE 27 DE DEZEMBRO DE 2002. 2002. Disponível em: < http://www.planalto.gov.br/CCIVIL/decreto/2002/D4553.htm >. Acesso em: 25 de Janeiro de 2009.

BRASIL. LEI No 11.907, DE 2 DE FEVEREIRO DE 2009. 2009d. Disponível em: < https://www.planalto.gov.br/ccivil_03/_Ato2007-2010/2009/Lei/L11907.htm >. Acesso em: 22 de Julho de 2009.

BRASIL. TCU - Tribunal de Contas da União. Levantamento acerca da Governança de Tecnologia da Informação na Administração Pública Federal. Relator Ministro Benjamin Zymler. Brasília: TCU, SEFTI - Secretaria de Fiscalização de Tecnologia da Informação, 2008d.

BRASIL. TCU - Tribunal de Contas da União. Levantamento acerca da Governança de Tecnologia da Informação na Administração Pública Federal. Relator Ministro Aroldo Cedraz. Brasília: TCU, SEFTI - Secretaria de Fiscalização de Tecnologia da Informação, 2010.

CASSIDY, A. A Pratical Guide to Information Systems Strategic Planning. 2. ed. USA: Auerbach Publications, 2005.

CBP - Center of Business Practices. Project Management Maturity: a benchmark of current best practices. USA. 2006. Disponível em: < http://www.cbponline.com/ >. Acesso em: 10 Julho de 2007.

CERVO, A. L.; BERVIAN, P. A.; SILVA, R. Metodologia Científica. 6. ed. São Paulo: Pearson Prentice Hall, 2007.

CRAWFORD, J. K. Project Management Maturity Model (CBP - Center for Business Practices). 2. ed. USA: Auerbach Publications, 2007.

CURRY, J.; FERGUSON, J. Increasing the Success of the Information Technology Strategic Planning Procces. 33rd Hawaii International Conference on System Sciences, 2000.

DYBA, T.; KAMPENES, V.; SJOBERG, D. A Systematic Review of Statistical Power in Software Engineering Experiments. Journal of Information and Software Technology, v. 1, n. 11, 2005.

GALLIERS, R. D.; LEIDNER, D. E. Strategic Information Management: challenges and strategies in managing information systems. 4. ed. USA: Routledge, 2009. 
GIL, A. C. Métodos e técnicas de pesquisa social. 6. ed. São Paulo: Atlas, 2009.

GOYAL, D. P. Information Systems Planning Practices in Indian Public Enterprises. Information Management \& Computer Security, v. 15, n. 3, p. 201-213, 2007.

GROVER, V.; SEGARS, A. An Empirical Evaluation of Stages of Strategic Information Systems Planning: patterns of process design and effectiveness. Information \& Management, v. 42, p. 761-779, 2005.

HONG, E. K. Information Technology Strategic Planning. IEEE Computer Society, 2009.

IRANI, Z. Information Systems Evaluation: navigating through the problem domain. Information \& Management, v. 40, p. 11-24, 2002.

ISO/IEC 12207. International Organization for Standardization / International Electrotechnical Comission. Systems and Software Engineering - Software Life Cycle Processes, Geneve: ISO, 2008.

ISO/IEC 15504-1. International Organization for Standardization / International Electrotechnical Comission. Information Technology - Process Assessment - Part 1: concepts and vocabulary, Geneve: ISO, 2004.

ITGI - IT Governance Institute. COBIT - Framework Control Objectives Management Guidelines Maturity Models. Versão.1. USA: ITGI, 2007.

KERZNER, H. Project Management Best Practices: achieving global excellence. 1. ed. USA: John Wiley \& Sons, 2006.

KERZNER, H. Using the Project Management Maturity Model: strategic planning for project management. 2. ed. USA: John Wiley \& Sons, 2005.

KITCHENHAM, B. A.; DYBA, T.; JORGENSEN, M. Evidence-based Software Engineering. Proceedings of the 26th International Conference on Software Engineering (ICSE' 04), p. 273-281, 2004.

KITCHENHAM, B. A.; MENDES, E.; TRAVASSOS, G. H. Cross Versus within Company Cost Estimation Studies: a systematic review. IEEE Transactions on Software Engineering, v. 33, n. 5, p. 316-329, 2007.

KUNNATHUR, A. S.; SHI, Z. An Investigation of The Strategic Information Systems Planning Success in Chinese Publicly Traded Firms. International Journal of Information Management, v. 21, p. 423-439, 2001.

LAKATOS, E. M.; MARCONI, M. A. Metodologia científica. 5. ed. São Paulo: Atlas, 2009.

LI, M.; YE, L. R. Information Technology and Firm Performance: linking with environmental, strategic and managerial contexts. Information \& Management, v. 35, p. 43-51, 1999.

LITTELL J. H.; CORCORAN J.; PILLAI, V. Systematic Reviews and Meta-analysis. USA: Oxford University Press, 2008. 
LUFTMAN, J.; KEMPAIAH, R.; NASH, E. Key Issues for IT Executives 2005. MIS Quarterly Executive, v. 5, n. 2, p. 27-45, 2005.

MAFRA, S. N.; TRAVASSOS, G. H. Estudos Primários e Secundários apoiando a busca por Evidência em Engenharia de Software. Relatório Técnico RT-ES687/06, Programa de Engenharia de Sistemas e Computação (PESC), COPPE/UFRJ, 2006.

MENTZAS, G. Implementing an IS Strategy - A Team Approach. Long Range Planning, v. 30, n. 1, p. 84-95, 1997.

NEWKIRK, H. E.; LEDERER, A. L. The effectiveness of strategic information systems planning under environmental uncertainty. Information \& Management, v. 43, p. 481-501, 2006.

OGC - Office of Government Commerce. Portfolio, Programme and Project Management Maturity Model (P3M3). Versão 2.0. London: OGC, 2008.

OGC - Office of Government Commerce. PRINCE2 Maturity Model (P2MM). Versão 1.0. London: OGC, 2006.

PAI, M.; MCCULLOCH, M.; GORMAN, J.; PAI, N.; ENANORIA, W.; KENNEDY, G.; THARYAN, P.; COLFORD JR., J. Systematic reviews and meta-analyses: An illustrated step-by-step guide. The National Medical Journal of India, v. 17, n. 2, p. 86-95, 2004.

PENNYPACKER, J. S. Project Portfolio Management Maturity Model. USA: CBP - Center for Business Practices, 2005.

PEREZ, B. E. Information Systems Investment Decisions in Business Practice: the spanish case. European Journal of Information Systems, v. 7, p. 202-209, 1998.

PHILIP, G. IS Strategic Planning for Operational Efficiency. Information Systems Management, v. 24, p. 247-264, 2007.

PMI - Project Management Institute. How Mature is Your Organization - Really? PM Network, v. 23. n. 2, 2009. ISSN 1040-8754.

PMI - Project Management Institute. Organization Project Management Maturity Model (OPM3). Newton Square: PMI, 2003.

PRADO, D. S. Maturidade em Gerenciamento de Projetos. Nova Lima: INDG Tecnologia e Serviços Ltda., 2008.

REZENDE, D. A. Planejamento de Informações Públicas Municipais: sistemas de informação e de conhecimento, informática e governo eletrônico integrados aos planejamentos das prefeituras e municípios. RAP, v. 41, n. 3, p. 505-536, 2007b.

REZENDE, D. A. Planejamento de Sistemas de Informação e Informática: guia prático para planejar a Tecnologia da Informação integrada ao planejamento estratégico das organizações. 2. ed. São Paulo: Atlas, 2007a.

SALMELA, H.; LEDERER, A.; REPONEN, T. Information Systems Planning in a Turbulent Environment. European Journal of Information Systems, v. 9, n. 1, p. 3-15, 2000. 
SANTOS, A. R. Metodologia Científica: a construção do conhecimento. 5. ed. Rio de Janeiro: DP\&A, 2002.

SEI - Software Engineering Institute. CMMI for Development: improving processes for better products. CMMI-DEV 1.2. Pittsburgh, PA: Carnegie Mellon University, 2006.

SOFTEX - Associação para Promoção da Excelência do Software Brasileiro. MPS.BR - Guia Geral, 2009. Disponível em: < http://www.softex.br/ >. Acesso em: 09 de Dezembro de 2010.

TEIXEIRA FILHO, J. G. A. MMPE-SI/TI (Gov) - Modelo de Maturidade para Planejamento Estratégico de SI/TI direcionado às Organizações Governamentais Brasileiras baseado em Melhores Práticas. vol. 1 e 2, 2010. Tese (Doutorado em Ciências da Computação) - Universidade Federal de Pernambuco (UFPE), Recife, 2010.

TEUBNER, R. A. Strategic Information Systems Planning: a case study from the financial services industry. Journal of Strategic Information Systems, v. 16, p. 105-125, 2007.

THONG, J. Y. L. An Integrated Model of Information Systems Adoption in Small Business. Journal of Management Information Systems, v. 15, n. 4, p. 187-214, 1999.

WARD, J.; PEPPARD, J. Strategic Planning for Information Systems. 3. ed. USA: John Wiley \& Sons, 2002. 